\title{
Long run Convergence in a Neo-Kaleckian Open-economy Model with Autonomous Export Growth
}

\author{
Won Jun Nah and Marc Lavoie
}

February 2016

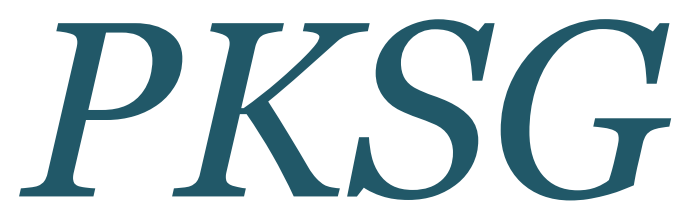

\section{Post Keynesian Economics Study Group}

\section{Working Paper 1604}

This paper may be downloaded free of charge from www.postkeynesian.net

(C) Won Jun Nah and Marc Lavoie

Users may download and/or print one copy to facilitate their private study or for non-commercial research and may forward the link to others for similar purposes. Users may not engage in further distribution of this material or use it for any profit-making activities or any other form of commercial gain. 


\title{
Long run Convergence in a Neo-Kaleckian Open-economy Model with Autonomous Export Growth
}

\begin{abstract}
A simple neo-Kaleckian open-economy model is presented and its implications for growth regimes are analyzed. The present model features long run-convergence to its normal rate of capacity utilization, which is conditionally achieved by incorporating the Harrodian principle of instability and autonomous growth in foreign demand. It is demonstrated that some aspects of the main Kaleckian results can be preserved not only in the short run but also in the long run, in the sense that both (i) a decrease in the propensity to save, and (ii) a change in income distribution favoring labor, bring about higher average rates of production growth and capital accumulation. However, the long-run impact of a change in the profit share is shown to be subjected to the condition that the responsiveness of the real exchange rate with respect to the profit share has to be bounded from above, confirming that the scope for wage-led demand or wage-led growth can be limited by open-economy considerations.
\end{abstract}

Keywords: neo-Kaleckian, growth, capacity utilization, exports, profit share, real exchange rate

JEL classifications: E11, F41, O41

\author{
Won Jun Nah \\ Associate Professor and Chair, School of Economics and Trade, Kyungpook National University, \\ Daegu, Korea \\ Marc Lavoie \\ Professor, Department of Economics, University of Ottawa, Ottawa, Canada \\ Address for correspondence: Marc Lavoie, mlavoie@uottawa.ca
}




\section{Introduction}

It is often said that a major characteristic of post-Keynesian economics is the assertion that effective demand is the key driver of economic activity, both in the short and the long run, and hence that the level and growth of productive capacity depends on aggregate demand. This is the so-called "Keynesian Hypothesis" - "the idea that investment is, in both the long run and the short run, independent of the savings that would be forthcoming from the normal utilisation of productive capacity" (Cesaratto 2015, p. 154). Traditionally, the argument has been justified on the grounds that either income distribution or the rate of utilization could be considered as endogenous variables. There has been a recent resurgence of interest in the argument, proposed by Franklin Serrano (1995a, 1995b) and Heinrich Bortis (1997, ch. 4), that the Keynesian Hypothesis could be sustained instead through the endogeneity of the share in GDP of an autonomous component of aggregate expenditure. This argument has been called the Sraffian supermultiplier view since its proponents were Sraffian authors, who argued, as had Pierangelo Garegnani (2015 [1964]), that investment was best viewed as a component of induced demand, reacting to the evolution of sales. Over the last few years, there has been a number of theoretical contributions taking up this version of the Keynesian Hypothesis, namely by Fabio Freitas and Serrano himself (2015) as well as Sergio Cesaratto (2015). The role of an exogenous demand component growing at an exogenous rate within an otherwise neo-Kaleckian framework, which will be the approach taken here, is a new field of study that has been first proposed by Olivier Allain (2015), and then by Marc Lavoie (2016), Riccardo Pariboni (2015) and Amitava Dutt (2015).

The purpose of the present study is to pick up the supermultiplier argument of Serrano and extend it to a small open-economy framework. Whereas Serrano took as a given the growth rate of autonomous consumption expenditures, here we will assume that the growth rate of autonomous exports is the exogenous component of aggregate expenditures. This has an obvious similarity with the balance-of-payment constrained models which are so popular and which were first put forward by Anthony Thirlwall (1979) and developed in McCombie and Thirlwall (1994), where the growth rate of exports in a 
country is a given, determined by the growth rate of world demand. However, our version of Serrano's supermultiplier view will be based on the framework developed by neoKaleckian authors such as Rowthorn (1981), Taylor (1983) and Dutt (1984). There has also been a resurgence of studies developing various aspects of a neo-Kaleckian model of growth extended to an open economy. The first such model was that of Robert Blecker (1989), and it has been followed by contributions by Blecker $(1999,2011)$ again and by Hein and Vogel (2008). These kinds of models have been further extended to explicit twocountry models in a number of ways, namely by Anton Rezai (2015), von Arnim et al. (2014) as well as Capaldo and Izurieta (2013). Dutt (2015) is the first attempt at combining the Serrano approach with the neo-Kaleckian framework within an open-economy model. De Juan (2014) also introduces exports growing at an exogenous rate within a Serrano type of model.

A key feature of the present model is its convergence in the long run to the normal rate of capacity utilization. This is conditionally achieved by incorporating both the Harrodian principle of instability and an autonomous growth rate in external demand which arises from world income growth. It is demonstrated that a critical aspect of the main Kaleckian results can be preserved not only in the short run but also over time and finally in the long run. To focus on this issue while keeping our model as simple as possible, we introduce some inevitable simplifications. Firstly, as done by Allain (2015), we assume away interest payments, in our case those possibly arising from foreign debts. Secondly, functional income distribution is assumed to be exogenous. Thirdly, we consider only purely quantitative adjustments. Hence we do not consider the complications due to the possible ramifications that changes in the real exchange rate could have on income distribution.

Our paper will proceed as follows. First, we present the accounting and behavioural equations of our model within the context of a small open economy. Second, we examine what happens in the short run to the rate of utilization and the growth rate of the economy when there is a change in the propensity to save and in the profit share. With respect to the latter change, this means we will be examining under which conditions such a model 
economy, which has a wage-led domestic demand, in concordance with the empirical measures of Onaran and Galanis (2012) and Onaran and Obst (2016), remains wage-led despite being subjected to an external constraint. In the third section, we will deliberate on the dynamic properties of our model in the medium run, when autonomous export growth is introduced. The impacts of changes in the propensity to save and the share of profits are examined. In the fourth section, we will examine whether the model has long run dynamic stability or not, checking under what conditions dynamic stability is preserved despite the addition of a Harrodian instability mechanism that purports to achieve normal rates of capacity utilization in the long run. Also we will compare the medium-run versus long-run impacts of a change in the growth rate of exports. The last section concludes.

\section{The Economic Environment}

Following Hein and Vogel (2008), we assume an open economy which imports inputs or intermediate goods and then produces outputs for both domestic purposes and for exports. The international markets are assumed to be competitive in the sense that countries can change neither import prices nor export prices: in other words, there is a 100 per cent passthrough. ${ }^{1}$ The price setting power of domestic firms is limited due to international competition. We assume that the threat of relocation of production facilities by entrepreneurs is credible enough to hinder any possible change in domestic functional income distribution which is due to the wage setting power of labor unions, so that the income distribution between capital and labor is exogenously determined through the political process. We also assume that, for simplification purposes, there are no government activities.

In this open economy without a public sector, macroeconomic equilibrium can be characterized as the fundamental identity which dictates that saving $(S)$ should equal the sum of investment expenditures $(I)$ and net exports, the latter being the trade balance $T B$ :

\footnotetext{
${ }^{1}$ This is obviously a simplification, although Coutts and Norman (2013) argue from past empirical studies that while finished goods are subjected to a partial pass-through in advanced economies, intermediate products, which are considered by firms as a cost, are usually subjected to a full pass-through.
} 


$$
S=I+T B=I+\left(X-e_{R} M\right) \text {. }
$$

The variable $e_{R}$ denotes the real exchange rate, which is, by definition, $e_{R}=e p_{f} / p_{d}$, where $e, p_{f}$ and $p_{d}$ are the nominal exchange rate, the foreign price level, and the domestic price level. $X$ and $M$ are real exports and imports. Out of the exports $X$, we separate the autonomously growing part $X_{0}$ from the (stationary) part $X_{1}$ which is a function of the real exchange rate, so that $X=X_{0}+X_{1}\left(e_{R}\right)$.

We employ the following linear specifications for investment expenditure and trade balance, both expressed in terms of the stock of capital. The investment function (2) below follows the standard neo-Kaleckian literature, where $g$ is the rate of accumulation of capital, $K$ the stock of capital, $u$ the rate of capacity utilization, $u_{n}$ the normal rate of capacity utilization, $\gamma$ the animal spirits of firms, and $\gamma_{u}>0$ a parameter which captures the sensitivity of the rate of accumulation to changes in the rate of utilization. Note that if capacity utilization is at its normal or desired rate, then $g=\gamma$, and one would think that the rate of accumulation desired by firms corresponds to the expected growth rate of sales, so as to keep the rate of utilization at its target level, as was pointed out by Committeri (1986, p. 173) and Caserta (1990, p. 152).

$$
\begin{gathered}
g=I / K=\gamma+\gamma_{u}\left(u-u_{n}\right) \\
t b=T B / K=x+\phi_{e} e_{R}-\phi_{u} u
\end{gathered}
$$

The net export function (3) deserves some attention. Here the autonomous component of exports is represented by $x=X_{0} / K$. The other components of net exports are assumed to be increasing in the real exchange rate and decreasing in the domestic demand, which is $\left(X_{1}-e_{R} M\right) / K=\phi_{e} e_{R}-\phi_{u} u$, where $\phi_{e}$ and $\phi_{u}$ are both positive.

Importantly, we assume that $X_{0}$ is exogenously increasing at the constant rate $\bar{g}_{x}>$ 0 , along with world income growth. In essence, we are considering the situation where the model economy is too small to affect the rate of world income growth and thus we take the growth rate of autonomous exports, driven by world income growth, as a given. In this small economy, the exogenous and continuous growth in foreign demand becomes the 
driving force for sustained growth, as we shall see. In this vein, Kaldor (1970) has been explicit on the importance of the rate of export growth in the growth of national output. He claimed that "from the point of view of any particular region, the 'autonomous component of demand' is the demand emanating from outside the region; ... the rate of economic development of a region is fundamentally governed by the rate of its exports" (Kaldor 1970, p. 342). It is in this regard that the global developments in effective demand may matter for the growth rate of an individual country in the long run.

The effects on net exports due to changes in the real exchange rate and domestic demand are reflected in the parameters $\phi_{e}$ and $\phi_{u}$, respectively. We assume that both are strictly positive. Positivity of $\phi_{u}$ is a standard assumption in that imports increase more often than not when domestic demand expands. The assumption that $\phi_{e}>0$ is necessary for the Marshall-Lerner condition to hold so that real depreciation (appreciation) yields an increase (decrease) in net exports in this model economy.

Assuming away the possibility of saving out of wages, as is also standard in neoKaleckian models, we can normalize saving, which is on the far left-hand side of equation (1), by dividing it by the stock of capital $K$,

$$
S / K=s_{p} \pi u
$$

where $s_{p}$ denotes the propensity to save from capitalists' profits, $P$ is profits, $q$ is real income, $\pi=P / q$ is the share of profits out of national income, and $u=q / K$ is the proxy for the rate of capacity utilization. The equilibrium condition (1) thus can be rewritten as follows:

$$
s_{p} \pi u=\gamma+\gamma_{u}\left(u-u_{n}\right)+x+\phi_{e} e_{R}-\phi_{u} u
$$

\section{Short-run Consequences}

The utilization rate $u^{*}$ and the growth rate $g^{*}$ in the short run equilibrium are obtained from (4): 


$$
u^{*}=\frac{\gamma-\gamma_{u} u_{n}+x+\phi_{e} e_{R}}{s_{p} \pi-\gamma_{u}+\phi_{u}}
$$

and

$$
g^{*}=\gamma+\gamma_{u}\left(\frac{\gamma+x+\phi_{e} e_{R}-\left(s_{p} \pi+\phi_{u}\right) u_{n}}{s_{p} \pi-\gamma_{u}+\phi_{u}}\right)
$$

where superscript * denotes short run equilibrium values of endogenous variables. In the context of the present model, the term "short-run" can be understood as an instantaneous snapshot, before all level variables start growing away from their equilibrium values in a given short period, so that, in particular, the $x=X_{0} / K$ ratio is a given despite its two components growing at different rates, in general, in the short run.

To define this short-run equilibrium as a stable one, we assume that the Keynesian stability condition holds, i.e., saving should more strongly respond to the changes in the rate of utilization than the sum of investment and net exports:

$$
s_{p} \pi>\gamma_{u}-\phi_{u}
$$

Also, since the rate of utilization of capacity should be non-negative and smaller than unity, it is required that:

$$
0 \leq \gamma-\gamma_{u} u_{n}+x+\phi_{e} e_{R} \leq s_{p} \pi-\gamma_{u}+\phi_{u}
$$

for otherwise the rate of capacity utilization could not be the adjusting variable any more.

The paradox of saving, understood as concerning both the short-run equilibrium rate of utilization and the rate of capital accumulation remains valid. This can be easily confirmed by checking that the partial derivatives $\partial u^{*} / \partial s_{p}$ and $\partial g^{*} / \partial s_{p}$ are both negative. On the other hand, the effects of a change in the functional distribution of income between capital and labor are ambiguous, thus depending on the values taken by the parameters of 
the model. However, we can derive the condition under which redistribution of income in favor of labor yields increases in demand and in the growth rate in a straightforward manner.

Making use of equations (5) and (6), it follows that:

$$
\begin{gathered}
\frac{\partial u^{*}}{\partial \pi}=\frac{\phi_{e} \frac{\partial e_{R}}{\partial \pi}-s_{p} u^{*}}{s_{p} \pi-\gamma_{u}+\phi_{u}} \\
\frac{\partial g^{*}}{\partial \pi}=\gamma_{u} \frac{\partial u^{*}}{\partial \pi}
\end{gathered}
$$

Once again, from the above equation, it can easily be checked that the partial derivatives $\partial u^{*} / \partial \pi$ and $\partial g^{*} / \partial \pi$ are both negative if the short-run stability condition holds (the denominator is positive) while the numerator is negative. This will occur if and only if the following inequality holds:

$$
\frac{\partial e_{R}}{\partial \pi}<\frac{s_{p} u^{*}}{\phi_{e}}
$$

Plugging back the value of $u^{*}$ in equation (5), this implies the following inequality:

$$
\frac{\partial e_{R}}{\partial \pi}<\frac{s_{p}\left(\gamma-\gamma_{u} u_{n}+x+\phi_{e} e_{R}\right)}{\phi_{e}\left(s_{p} \pi-\gamma_{u}+\phi_{u}\right)}
$$

With an exogenous growth in exports, a redistribution of income towards wages results in a higher utilization rate of capacity and a higher accumulation rate of capital in the short run if inequality (9) is fulfilled.

The argument above implies that, for the model to generate a wage-led, rather than a profit-led, demand and growth regimes in the short run, the responsiveness of the real exchange rate with respect to a change in income distribution should be bounded from above. Open-economy considerations such as international competition seem to be able to limit the scope for wage-led demand and growth regimes. This result can be interpreted as 
being consistent with prevailing views in the Kaleckian literature, as found in Blecker (1989) and also Bhaduri and Marglin (1990).

Before moving on to the model dynamics, we summarize the relationship between the real exchange rate and income distribution in a brief fashion. As is well-known, the latter can fluctuate together with the change in the former via three different channels in the present model environment. These are changes in (i) the nominal exchange rate $e$, (ii) the real wage rate $w$, and (iii) the mark-up $\theta$. Out of these three channels through which $\pi$ affects $e_{R}$, the real exchange rate becomes positively associated with the profit share when changes in income distribution are due to changes in either $e$ or $w$. To see this, from the relation identified by Hein and Vogel (2008)

$$
\pi(e, w, \theta)=\frac{\theta(1+j(e, w))}{1+\theta(1+j(e, w))}
$$

Let us define an implicit function

$$
e_{R}=\frac{e p_{f} y}{w(1+\theta)(1+j(e, w))} \equiv e_{R}\left(\pi(e, w, \theta) ; p_{f}, y\right)
$$

where $y$ denotes labor productivity and $j=j(e, w)$ is the ratio of unit material costs to unit direct labor costs which increases in $e$ and decreases in $w$, i.e., $j_{e}>0$ and $j_{w}<0$. Recall that we are treating income distribution, hence the profit share $\pi$, as a purely exogenous variable which is political in its nature, and the value of which is realized together with $w$ and $\theta$, given $e$. Intuitively, if the price of imported material rises when $e$ goes up, then the share of wages should shrink, and thus $\pi$ should increase, while $e_{R}$ should rise. If $w$ goes up, other things being equal, then clearly $\pi$ should fall, while $e_{R}$ should also fall because domestic prices rise relative to foreign prices.

If, on the contrary, a change in $e_{R}$ is associated with a change in income distribution which comes about due to a change in $\theta$, then $e_{R}$ may respond negatively to $\pi$. Therefore, at least in theory, there seems to be an ambiguity in the relationship between the real 
exchange rate and income distribution. However, it is clearly documented by Hein (2014, p. 316) that "empirically, if there is any relationship between the profit share and international competitiveness, this relationship seems to be positive". Overall, it seems that we can confidently assume that real depreciation (appreciation) can be thought of as reflecting a corresponding rise (fall) in the profit share within the model environment of this paper.

\section{Medium-run Dynamics}

However, the short-run equilibrium given by equations (5) and (6) will get modified once $x$ starts to change over time. The autonomous component of export demand $X_{0}$ grows exogenously at the rate $\bar{g}_{x}$. As long as we stick to this exogenous growth component of effective demand within our model, it is helpful to distinguish conceptually between three different successive equilibria, which are the short-run, medium-run and long-run equilibria, as in Allain (2015). In the short run, $x$ is constant and hence the effects of the autonomous export growth cannot be considered. Contrarily, we define the medium run as being the period during which $X_{0}$ grows. Then the medium-run equilibrium can be defined as a point of rest where $x$ converges to a certain value. Finally, long-run issues will be discussed in the next section.

Considering the definition of $x$, we see that $x$ evolves through time as follows, where a hat on a variable denotes its instantaneous growth rate:

$$
\hat{x}=\bar{g}_{x}-g^{*}=\bar{g}_{x}-\gamma-\gamma_{u}\left(\frac{\gamma+x+\phi_{e} e_{R}-\left(s_{p} \pi+\phi_{u}\right) u_{n}}{s_{p} \pi-\gamma_{u}+\phi_{u}}\right)
$$

from which it is clear that:

$$
\frac{\partial \hat{x}}{\partial x}=-\frac{\gamma_{u}}{s_{p} \pi-\gamma_{u}+\phi_{u}}<0
$$


Assuming short-run stability, this implies that the behavior of $x$ is dynamically stable, and thus $x$ converges to its equilibrium value $x^{* *}$ in the medium run, where superscript $* *$ represents the medium-run equilibrium value of a variable.

The medium-run rate of accumulation is obtained by using equation (12) under the condition that $\hat{x}=0$ :

$$
g^{* *}=\bar{g}_{x}
$$

It follows from this that the medium-run rate of utilization becomes

$$
u^{* *}=u_{n}+\frac{\bar{g}_{x}-\gamma}{\gamma_{u}}
$$

since it should be that $g^{* *}=\gamma+\gamma_{u}\left(u^{* *}-u_{n}\right)$. Also, in the medium run equilibrium, the ratio of autonomous exports to capital stock, $x^{* *}$, is obtained from (12)

$$
x^{* *}=\left(s_{p} \pi+\phi_{u}\right) u^{* *}-\phi_{e} e_{R}-\bar{g}_{x}
$$

It seems natural that the next question be whether our model economy can or cannot successfully reproduce the standard Keynesian and/or Kaleckian results in the long run. We have already investigated the short run consequences of the model. It is shown that, in the short run, the paradox of saving is valid whereas the scope for wage-led growth gets narrower than in a closed economy environment. If it is the case, then what are the peculiar dynamic aspects of our model?

From equations (13) and (14), we find that, in the medium-run equilibrium, both the rates of accumulation and utilization are not affected by changes in the propensity to save or in income distribution. However, from equation (15), it is straightforward to see that $\partial x^{* *} / \partial s_{P}>0$. The higher the propensity to save, the lower the level of capital stock for a given amount of autonomous exports. An increase in the propensity to save does not affect the medium-run growth rate of the capital stock, but it decrease its level. This can be 
interpreted as implying that one of the key Kaleckian results is still valid in the medium run of our model.

We also can see that $\partial x^{* *} / \partial \pi>0$, under the condition that the following inequality holds. This inequality is exactly comparable to that given by (9) in the case of the short run.

$$
\frac{\partial e_{R}}{\partial \pi}<\frac{s_{p} u^{* *}}{\phi_{e}}=\frac{s_{p}}{\phi_{e}}\left(u_{n}+\frac{\bar{g}_{x}-\gamma}{\gamma_{u}}\right)
$$

How can we interpret the economic meaning of this inequality? Decomposing the change in $x^{* *}$ by totally differentiating (15), we have

$$
d x^{* *}=s_{p} u^{* *} d \pi-\phi_{e} \frac{\partial e_{R}}{\partial \pi} d \pi
$$

In the left hand side of the above equation, an increase in the share of profits has a twofold effect on capital accumulation. The first term captures a direct negative effect from decreases in consumption demand (due to income redistribution towards capitalists), while the second term captures an indirect positive effect from increases in net exports (due to the higher real exchange rate). The former dominates the latter if

$$
s_{p} u^{* *}-\phi_{e} \frac{\partial e_{R}}{\partial \pi}>0
$$

which is exactly equivalent to condition (16) above.

Unless the real exchange rate is overly sensitive to changes in income distribution, then any change in income distribution favoring labor brings about a higher level of capital stock for a given amount of autonomous exports. In other words, the average growth rate of the capital stock, i.e., the average accumulation rate, increases during the traverse following a decrease in the share of profits, until a new medium-run equilibrium is reached. It should be noted that this phenomenon should be more prominent when the propensity to save of capitalists is larger, or when the sensitivity of net exports to changes in the real exchange rate is lower. 
The discussions so far clearly show that our simplified open-economy model with autonomous export growth can be successful in generating the well-known Kaleckian results concerning both the relationship between growth and the propensity to save, and the one between growth and income distribution. This point can be illustrated with the help of Figure 1 and Figure 2.

Figure 1 exhibits the medium-run response of the hypothetical economy after a sudden rise in the propensity to save. The initial equilibrium is given at $\left(u_{0}^{* *}, g_{0}^{* *}\right)$ in the figure. Now suppose $s_{p}$ increases from $s_{p 0}$ to $s_{p 1}$. The instantaneous short run impact falls on the saving curve, rotating it counter-clockwise from $\sigma_{0,0}$ to $\sigma_{1,0}$. Thus, the economy moves from $\left(u_{0}^{* *}, g_{0}^{* *}\right)$ to $\left(u^{*}, g^{*}\right)$. As we have seen in the previous section, both the rates of accumulation and utilization decrease in the short run. ${ }^{2}$

However, that cannot be the whole story. Since the autonomous component of exports increases continuously at the constant rate of $\bar{g}_{x}$ while the rate of accumulation of capital slows down, the ratio $x$ must increase. Over time, $x$ increases from $x_{0}$ to $x_{1}$. This in turn results in a downward parallel shifts of the $\sigma$ curve in the upper panel from $\sigma_{1,0}$ to $\sigma_{1,1}$, and also to an upward parallel shift of the $(g+t b)$ curve in the lower panel from $g+$ $t b\left(x_{0}\right)$ to $g+t b\left(x_{1}\right)$.

\section{Figure 1 around here}

Now let us turn to the effects of a redistribution of functional income between workers and capitalists. Figure 2 compares the short-run and medium-run responses of the rate of accumulation and the rate of utilization of capacity after an income distribution

\footnotetext{
${ }^{2}$ In Figure 1 , we define $\sigma_{i, j} \equiv s_{p i} \pi u-t b\left(x_{j}\right)$. In the upper panel, the slopes of the $g$ curve and that of the $\sigma$ curve are $\gamma_{u}$ and $\left(s_{p} \pi+\phi_{u}\right)$, respectively. The values of the intercepts on the vertical axis are $(\gamma-$ $\left.\gamma_{u} u_{n}\right)$ and $-\left(x+\phi_{e} e_{R}\right)$, respectively. On the other hand, in the lower panel, the slopes of the $(g+$ $t b)$ curve and that of the $s_{p} \pi u$ curve are $\left(\gamma_{u}-\phi_{u}\right)$ and $s_{p} \pi$, respectively. The values of the vertical intercepts are $\left(\gamma-\gamma_{u} u_{n}+x+\phi_{e} e_{R}\right)$ and 0 , respectively.
} 
shock, which favors capital. Here, recall that we are implicitly assuming that the sensitivity of the real exchange rate with respect to changes in income distribution is small enough, the inequalities given by equations (9) and (16) being assumed to be fulfilled.

\section{Figure 2 around here}

This time, let us suppose that the share of profits $\pi$ goes up from $\pi_{0}$ to $\pi_{1}$. In the short run, the saving curve rotates and shifts down from $\sigma_{0,0}$ to $\sigma_{1,0}$ in the upper panel. Also, the $(g+t b)$ curve in the lower panel shifts up, reflecting the effects of an increase in the real exchange rate on the trade balance. Hence, in the short run, the economy moves to $\left(u^{*}, g^{*}\right) \cdot{ }^{3}$ Again over time, the ratio $x$ increases from $x_{0}$ to $x_{1}$. This yields further downward parallel shifts of the $\sigma$ curve in the upper panel from $\sigma_{1,0}$ to $\sigma_{1,1}$, and at the same time further upward parallel shifts of the $(g+t b)$ curve in the lower panel, from $g+$ $t b\left(\pi_{1}, x_{0}\right)$ to $g+t b\left(\pi_{1}, x_{1}\right)$. All of these shifts continue until the economy reaches $\left(u_{1}^{* *}, g_{1}^{* *}\right)$, which is the new medium-run equilibrium where the evolution of $x$ stops.

Figure 3 summarizes the responses of some important endogenous variables to a permanent increase in the propensity to save and in the share of profits over the short run and the medium run. Considering the whole traverse of variables over the medium run, and comparing their average rates of growth, we can claim that the main Kaleckian results are still (conditionally) valid, even when we introduce autonomous growth in exports into an otherwise canonical open-economy neo-Kaleckian model.

\section{Figure 3 around here}

\footnotetext{
${ }^{3}$ In Figure 2, we define $\sigma_{i, j} \equiv s_{p} \pi_{i} u-t b\left(\pi_{i}, x_{j}\right)$. All the values of slopes and intercepts are the same as in Figure 1.
} 


\section{Long-run Adjustments with Harrodian Mechanism}

From an empirical point of view, the medium-run equilibrium defined by equations (13) and (14) can be subjected to criticism, since it is known that the rate of utilization of capacity exhibits strong stationarity. The equilibrium rate of utilization given by equation (13) may not coincide with its normal or desired rate. Hence, we need a mechanism that will bring the equilibrium rate of capacity utilization, $u^{* *}$, towards the normal rate of capacity utilization, $u_{n}$, over a longer horizon than the medium-run we have defined.

As was already clearly spelled out, in the present model the long-run adjustments of the rate of utilization toward its normal rate is driven by the action of the famous Harrodian instability mechanism. It seems unrealistic to assume that the growth rate of sales expected by firms, which is captured by the parameter $\gamma$ in the investment function, stays at the same value forever. Over time, it should slowly adjust to past changes in the growth rate of sales. In this regard, we can assume that $\gamma$, which so far, in the medium run, was considered as an exogenous constant, will begin to adjust. This mechanism was first proposed by Allain (2015) within the context of a neo-Kaleckian growth model with an autonomous non-capacity generating demand component growing at an exogenous rate. Using the slightly modified version proposed by Lavoie $(2014,2016)$, the mechanism can be reformulated as the following Harrodian equation:

$$
\hat{\gamma}=\Psi_{0}\left(u^{* *}-u_{n}\right)
$$

with $\Psi_{0}>0$. This appeals to intuition in that, if the actual rate of utilization exceeds the desired or normal rate, then equation (2) implies that the growth rate of the economy exceeds the expected growth rate of sales, so that firms ought to revise upwards their expectations of future sales growth. Now using (5), this equation becomes:

$$
\hat{\gamma}=\Psi_{0}\left(\frac{\bar{g}_{x}-\gamma}{\gamma_{u}}\right)
$$


from which we can easily see that this long-run adjustment process becomes in effect stable. This stability is due to the interactive effects of the Harrodian mechanism with the growth of the autonomous demand components. ${ }^{4}$

$$
\frac{\partial \hat{\gamma}}{\partial \gamma}=-\frac{\Psi_{0}}{\gamma_{u}}<0
$$

The long run equilibrium of the model is achieved when $\hat{\gamma}=0$. Thus, it is described as follows:

$$
\begin{aligned}
& g^{* * *}=\bar{g}_{x} \\
& u^{* * *}=u_{n}
\end{aligned}
$$

and plugging (19) into the equation (15), we also have:

$$
x^{* * *}=\left(s_{p} \pi+\phi_{u}\right) u_{n}-\phi_{e} e_{R}-\bar{g}_{x}
$$

Obviously, as also noted by Lavoie (2016), the growth rate of autonomous expenditures, here $\bar{g}_{x}$, cannot be too high otherwise the solutions of the autonomous exports to capital ratio, $x^{* * *}$ and $x^{* *}$, would be negative, which would seem to be meaningless.

It should be noted that we finally reached a long-run equilibrium where the rate of utilization of capacity converges to its normal, or desired rate, in the context of an openeconomy neo-Kaleckian growth model. The task has been achieved by introducing both the autonomous growth of foreign aggregate demand and the Harrodian instability mechanism. We believe that it is plausible to assume that the sustained growth of a small open economy critically hinges on the evolution of global effective demand. The autonomous growth in aggregate demand emanating from the external sector facilitates domestic growth in the long run - a point also made by the authors of Kaldorian growth models, as developed by McCombie and Thirlwall (1994). Moreover, together with the Harrodian mechanism, this growth component can contribute to bringing the economy into

\footnotetext{
${ }^{4}$ In fact, this is because the instability inherent to the Harrodian mechanism becomes more than offset by the exogenous growth of effective demand. The argument will become more elaborate in the Appendix of this paper.
} 
a fully-adjusted position. This view is in a stark opposition to the traditional belief that long-run economic growth depends solely on supply-side developments.

From equations (18) and (19), it is evident that changes in neither the propensity to save nor the share of profits can affect the rates of accumulation or of capacity utilization in the long run. However, from (20) we have $\partial x^{* * *} / \partial s_{P}>0$, and $\partial x^{* * *} / \partial \pi>0$ under the following inequality condition, which is simply a long-run version of equations (9) or (16).

$$
\frac{\partial e_{R}}{\partial \pi}<\frac{s_{p} u_{n}}{\phi_{e}}
$$

The long-run impact of a change in income distribution is subjected to the condition that the sensitivity of the real exchange rate to changes in the profit share has to be bounded from above, with this upper bound depending on both the propensity to save and the responsiveness of net exports to the real exchange rate.

Once we assume that the hypothetical model economy starts from a fully-adjusted position with the actual rate of capacity utilization at $u_{n}$, that is, starting from an initial long-run equilibrium, then both a decrease in the propensity to save of capitalists and a redistribution of income favoring labor can be accompanied by a higher level of capital stock, a higher average rate of growth, and a higher average rate of utilization during the whole traverse until a new long-run equilibrium is reached. In this regard, most of the results obtained from our analyses dealing with the medium-run dynamics are maintained in the case of the longer run.

An additional experiment that requires some attention is the case where the exogenous growth rate of autonomous exports changes. Let us suppose that $\bar{g}_{x}$ increases from $\bar{g}_{x}^{0}$ to $\bar{g}_{x}^{1}$. Now $X_{0}$ grows at faster rate. What will happen in this case? Most notably, it is clear from equation (18) that the rate of accumulation of capital will be higher in the long run. Also, from equation (20) we have: 


$$
\frac{\partial x^{* * *}}{\partial \bar{g}_{x}}<0
$$

which means that the faster growth of the autonomous demand component will be accompanied by an even faster rate of capital accumulation in the long run.

But, from equation (15), and making use of equation (14), we can see that due to the Keynesian stability condition given in (7), we arrive at the following consequence for the autonomous exports to capital ratio when looking at the medium-run solution:

$$
\frac{\partial x^{* *}}{\partial \bar{g}_{x}}=\left(s_{p} \pi+\phi_{u}\right) \frac{\partial u^{* *}}{\partial \bar{g}_{x}}-1=\frac{s_{p} \pi-\left(\gamma_{u}-\phi_{u}\right)}{\gamma_{u}}>0
$$

An increase in $\bar{g}_{x}$ leads to a decrease of $x$ in the long run, but it increases $x$ in the medium run. ${ }^{5}$ How can these seemingly contradictory observations be reconciled? The discussions so far reveal that in the long run the faster growth rate of the autonomous demand component results in a permanent expansionary effect on the economy. However, for this long-run expansionary effect to occur, at least for a while, the growth rate of autonomous demand should surpass the rate of capital accumulation. This is what happens in the transition towards the medium run, along with the increase in the rate of capacity utilization which induces the increase in the pace of capital accumulation. Otherwise, i.e., if at all times aggregate demand increases more slowly or no faster than productive capacity, then there will not be any impetus for an induced acceleration of capital accumulation. Over the longer time horizon, during the transition towards the long run, through the Harrodian mechanism as entrepreneurs raise the expected growth rate of sales and adjust their investment decisions in view of their revised long-run forecasts, the rate of capital accumulation overcomes for a while the growth rate of sales and of autonomous demand. This brings down the rate of utilization back to its normal level while simultaneously also bringing down the $x$ ratio - the ratio of autonomous exports to capital, until finally the growth rate of capital and the growth rate of autonomous exports are brought back to equality in the long-run equilibrium.

\footnotetext{
${ }^{5}$ A similar result is obtained in Lavoie (2016, p. 15)
} 


\section{Concluding Remarks}

In this paper, we have embedded the Sraffian supermultiplier argument of Serrano into an otherwise canonical open-economy neo-Kaleckian framework. The role of an autonomous component of export growth, which is determined by the growth in world demand, has been explored from a small open-economy perspective. The model has been shown to be conditionally successful in reproducing some aspects of the main Kaleckian results, not only in the short run but also over time. An increase in the propensity to save or in the share of profits can decrease the average rates of capital accumulation and economic growth. We also confirm that the scope for wage-led demand or wage-led growth can be limited by the sensitivity of the real exchange rate to changes in income distribution.

It should be noted that we have employed as simple a reduced-form model as is possible to clarify our findings. One thing that would need further consideration in our model is that the trade balance is not likely to be in equilibrium even in the long run. In fact, the ratio of the long-run trade balance to the stock of capital is constant and

exogenously given at $t b^{* * *}=s_{p} \pi u_{n}-\bar{g}_{x}$, as can be easily deduced from equation (20). If our model economy was not growing but was in a stationary state instead, a permanent trade deficit would not be sustainable in the long run. However, in a constantly growing economy, this trade imbalance can be sustainable as long as it is increasing proportionally to economic expansion. This argument seems to be supported by real-life empirical observations.

We believe the discussions and analyses presented in this paper can be enriched within a more realistic model environment by extending the paper in at least three dimensions. Firstly and foremost, we need to consider explicitly the interest payments possibly arising from foreign debts or from foreign assets. Secondly, the possible repercussions of changes in the real exchange rate on income distribution would need to be addressed. Thirdly, the government sector would need to be added to our open-economy environment. 


\section{References}

Allain, O. (2015), 'Tackling the instability of growth: a Kaleckian-Harrodian model with an autonomous expenditure component', Cambridge Journal of Economics, 39, 1351-1371.

Bhaduri, A. and S. Marglin (1990), 'Unemployment and the real wage: the economic basis for contesting political ideologies', Cambridge Journal of Economics, 14 (4), December, 375-393.

Blecker, R.A. (1989), 'International competition, income distribution and economic growth', Cambridge Journal of Economics, 13 (3), September, 395-412.

Blecker, R.A. (2011), 'Open economy models of distribution and growth', in E. Hein and E. Stockhammer (eds), A Modern Guide to Keynesian Macroeconomics and Economic Policies, Cheltenham: Edward Elgar, pp. 215-239.

Bortis, H. (1997), Institutions, Behaviour and Economic Theory: A Contribution to ClassicalKeynesian Political Economy, Cambridge: Cambridge University Press.

Capaldo, J. and A. Izurieta (2013), 'The imprudence of labour market flexibilization in a fiscally austere world', International Labour Review, 152 (1), March, 1-26.

Caserta, M. (1990), 'The steady-state model of capital utilisation: a comment' Studi Economici, 41 (2), 139-153.

Cesaratto, S. (2015), 'Neo-Kaleckian and Sraffian controversies on the theory of accumulation', Review of Political Economy, 27 (2), April, 154-182.

Committeri, M. (1986), 'Some comments on recent contributions on capital accumulation, income distribution and capacity utilization', Political Economy: Studies in the Surplus Approach, 2 (2), 161-186.

Coutts, K and N. Norman (2013), 'Post-Keynesian approaches to industrial pricing: a survey and critique', in G.C. Harcourt and P. Kriesler (eds), Oxford Handbook of Post-Keynesian Economics, volume 1, Oxford: Oxford University Press, pp. 443-466.

De Juan, O. (2014), 'How to escape from the trap of the warranted rate of growth', working paper, Department of Economics and Finance, Universidad de Castilla-La Mancha, DT-DAET 2014/1.

Dutt, A. K. (2015), 'Growth and distribution with exogenous autonomous demand growth and normal capacity utilization', mimeo.

Freitas, F. and F. Serrano (2015), 'Growth, distribution and effective demand: the supermultiplier growth model alternative', Review of Political Economy, 27 (forthcoming). 
Garegnani, P. (2015), 'The problem of effective demand in Italian economic development: on the factors that determine the volume of investment', Review of Political Economy, 27 (2), April, 111-133. [Translation of a 1964 chapter in Italian].

Hein, E. (2014), Distribution and Growth After Keynes: A Post Keynesian Guide, Cheltenham: Edward Elgar.

Hein, E. and L. Vogel (2008), 'Distribution and growth reconsidered: Empirical results for six OECD countries', Cambridge Journal of Economics, 32 (3), May, 479-511.

Kaldor, N. (1970), 'The case for regional policies', Scottish Journal of Political Economy, 17 (3), November, 337-348.

Lavoie, M. (2014), Post-Keynesian Economics: New Foundations, Cheltenham: Edward Elgar.

Lavoie, M. (2016), 'Convergence towards the normal rate of capacity utilization in neo-Kaleckian models: The role of non-capacity creating autonomous expenditures', Metroeconomica, 67 (1), 172-201.

McCombie, J.S.L. and A.P. Thirlwall (1994), Economic Growth and the Balance-of-Payments Constraint, London: Macmillan.

Onaran, Ö. and G. Galanis (2012). 'Is aggregate demand wage-led or profit-led: national and global effects', working paper \# 40, Conditions of Work and Employment Series, International Labour Office.

Onaran, Ö. and T. Obst (2016). 'Wage-led growth in the EU15 member states: The effects of income distribution on growth, investment, trade balance, and inflation', Post-Keynesian Economics Study Group, PKSG1602, https://www.postkeynesian.net/working-papers/1602/

Pariboni, R. (2015), 'Autonomous demand and the Marglin-Bhaduri model: a critical note', Working paper 715, Department of Economia Politica e Statistica, University of Sienna, http://www.deps.unisi.it/sites/st02/files/allegatiparagrafo/26-09-2015/715.pdf

Rezai, A. (2015), 'Demand and distribution in integrated economies', Cambridge Journal of Economics, 39 (5), 1399-1414.

Rowthorn, B. (1981), 'Demand, real wages and economic growth', Thames Papers in Political Economy, Autumn, 1-39.

Serrano, F. (1995a), 'Long period effective demand and the Sraffian supermultiplier', Contributions to Political Economy, 14, 67-90.

Serrano, F. (1995b), The Sraffian Multiplier, PhD dissertation, Faculty of Economics and Politics, University of Cambridge. 
Taylor, L. (1983), Structuralist Macroeconomics: Applicable Models for the Third World, New York: Basic Books.

Thirlwall, A.P. (1979), 'The balance of payments constraint as an explanation of international growth rate differences', Banca Nazionale del Lavoro Quarterly Review, 32 (128), March, 45-53.

Von Arnim, R., D. Tavani and L. Barbosa de Carvalho (2014), 'Redisribution in a two-country neo-Kaleckian model', Metroeconomica, 65 (3), 430-459. 


\section{Appendix}

In the text of this paper, we have distinguished the medium run from the long run. If we do not make this distinction, then the Harrodian equation (17) can be rewritten as follows.

$$
\hat{\gamma}=\Psi_{1}\left(u^{*}-u_{n}\right)=\Psi_{1}\left(\frac{\gamma+x+\phi_{e} e_{R}-\left(s_{p} \pi+\phi_{u}\right) u_{n}}{s_{p} \pi-\gamma_{u}+\phi_{u}}\right)
$$

from which we can easily see that

$$
\frac{\partial \hat{\gamma}}{\partial \gamma}=\frac{\Psi_{1}}{s_{p} \pi-\gamma_{u}+\phi_{u}}>0
$$

The stability of the long-run equilibrium in the model can be verified by examining the properties of the following Jacobian matrix $J$, which represents the system of linear dynamic equations, (12) and (17A).

$$
J=\left[\begin{array}{ll}
\frac{\partial \hat{\gamma}}{\partial \gamma} & \frac{\partial \hat{\gamma}}{\partial x} \\
\frac{\partial \hat{x}}{\partial \gamma} & \frac{\partial \hat{x}}{\partial x}
\end{array}\right]=\left[\begin{array}{cc}
\frac{\Psi_{1}}{s_{p} \pi-\gamma_{u}+\phi_{u}} & \frac{\Psi_{1}}{s_{p} \pi-\gamma_{u}+\phi_{u}} \\
\frac{-\left(s_{p} \pi+\phi_{u}\right)}{s_{p} \pi-\gamma_{u}+\phi_{u}} & \frac{-\gamma_{u}}{s_{p} \pi-\gamma_{u}+\phi_{u}}
\end{array}\right]
$$

The determinant of this matrix, Det $J$, and its trace, $\operatorname{Tr} J$, are given by

$$
\begin{aligned}
& \text { Det } J=\frac{\Psi_{1}}{s_{p} \pi-\gamma_{u}+\phi_{u}} \\
& \operatorname{Tr} J=\frac{\Psi_{1}-\gamma_{u}}{s_{p} \pi-\gamma_{u}+\phi_{u}}
\end{aligned}
$$

This shows that the determinant is positive due to (7) and the trace can be negative if $\gamma_{u}>$ $\Psi_{1}$, i.e., if the effect of Harrodian instability is properly tamed. Hence we can say that the long-run equilibrium that the present system of dynamic equations produces is conditionally stable. This can be illustrated with the help of the phase diagram drawn in Figure A1. 
There are two demarcation lines which correspond to $\hat{\gamma}=0$ and $\hat{x}=0$, respectively.

$\hat{\gamma}=0$ :

$$
\gamma=-x+\left(s_{p} \pi+\phi_{u}\right) u_{n}-\phi_{e} e_{R}
$$

$\hat{x}=0$ :

$$
\gamma=-\frac{\gamma_{u}}{s_{p} \pi+\phi_{u}} x+\left(1-\frac{\gamma_{u}}{s_{p} \pi+\phi_{u}}\right) \bar{g}_{x}-\frac{\gamma_{u} \phi_{e} e_{R}}{s_{p} \pi+\phi_{u}}+\gamma_{u} u_{n}
$$

Figure Al around here

Clearly the long-run equilibrium is any combination of $\gamma$ and $x$ with $\hat{\gamma}=\hat{x}=0$, which is thus at the intersection of the two demarcation lines, (A1) and (A2). However, the latter $(\hat{x}=0)$ must be flatter than the former $(\hat{\gamma}=0)$ in the $(x, \gamma)$ plane, since the Keynesian stability condition (7) should hold. This guarantees uniqueness of the long-run equilibrium in the model. Also it is fairly straightforward to verify that the $x$-intercept of the line (A2) is larger than that of the line (A1) thanks to condition (7) again. Therefore, for the long-run equilibrium to be meaningfully defined, i.e., for the intersection of these lines to lie in the first quadrant such that both $\gamma^{* * *}=\bar{g}_{x}$ and $x^{* * *}$ become positive, it suffices that the $\gamma$-intercept of the line (A1) $\hat{\gamma}=0$ should be located higher than that of the line (A2) $\hat{x}=0$, i.e.,

$$
\left(s_{p} \pi+\phi_{u}\right) u_{n}-\phi_{e} e_{R}>\left(1-\frac{\gamma_{u}}{s_{p} \pi+\phi_{u}}\right) \bar{g}_{x}-\frac{\gamma_{u} \phi_{e} e_{R}}{s_{p} \pi+\phi_{u}}+\gamma_{u} u_{n}
$$

But, indeed, this sufficient condition simply reduces to 


$$
x^{* * *}>0
$$

which holds by assumption.

In Figure A2, the long-run equilibrium is the point $E$ along the line $\hat{\gamma}=0$, where $\gamma=\bar{g}_{x}$. From equation (A1), we can see that the line $\hat{\gamma}=0$ shifts up with a larger value of $s_{p}$, resulting in an increase in the long-run equilibrium value of $x^{* * *}$, which moves from $x_{0}^{* * *}$ to $x_{1}^{* * *}$. This implies that the average rate of capital accumulation becomes slower for a given rate of growth of autonomous exports.

\section{Figure A2 around here}

We can consider the case where $\pi$ increases. This time, the line $\hat{\gamma}=0$ may shift up or down, depending on the values of some relevant parameters. However under the assumption given by condition (21) in the main text, we can conclude that the line $\hat{\gamma}=0$ shifts up with a larger $\pi$, resulting in a slowdown of the average rate of capital accumulation.

Finally, we can also consider the case where $\bar{g}_{x}$ increases. Looking at equation (A2), we know that the line $\hat{x}=0$ should shift up with a higher $\bar{g}_{x}$, implying that $\partial x^{* * *} / \partial \bar{g}_{x}<$ 0 . This is illustrated in Figure A3, which clearly supports the claims of the text.

Figure A3 around here 


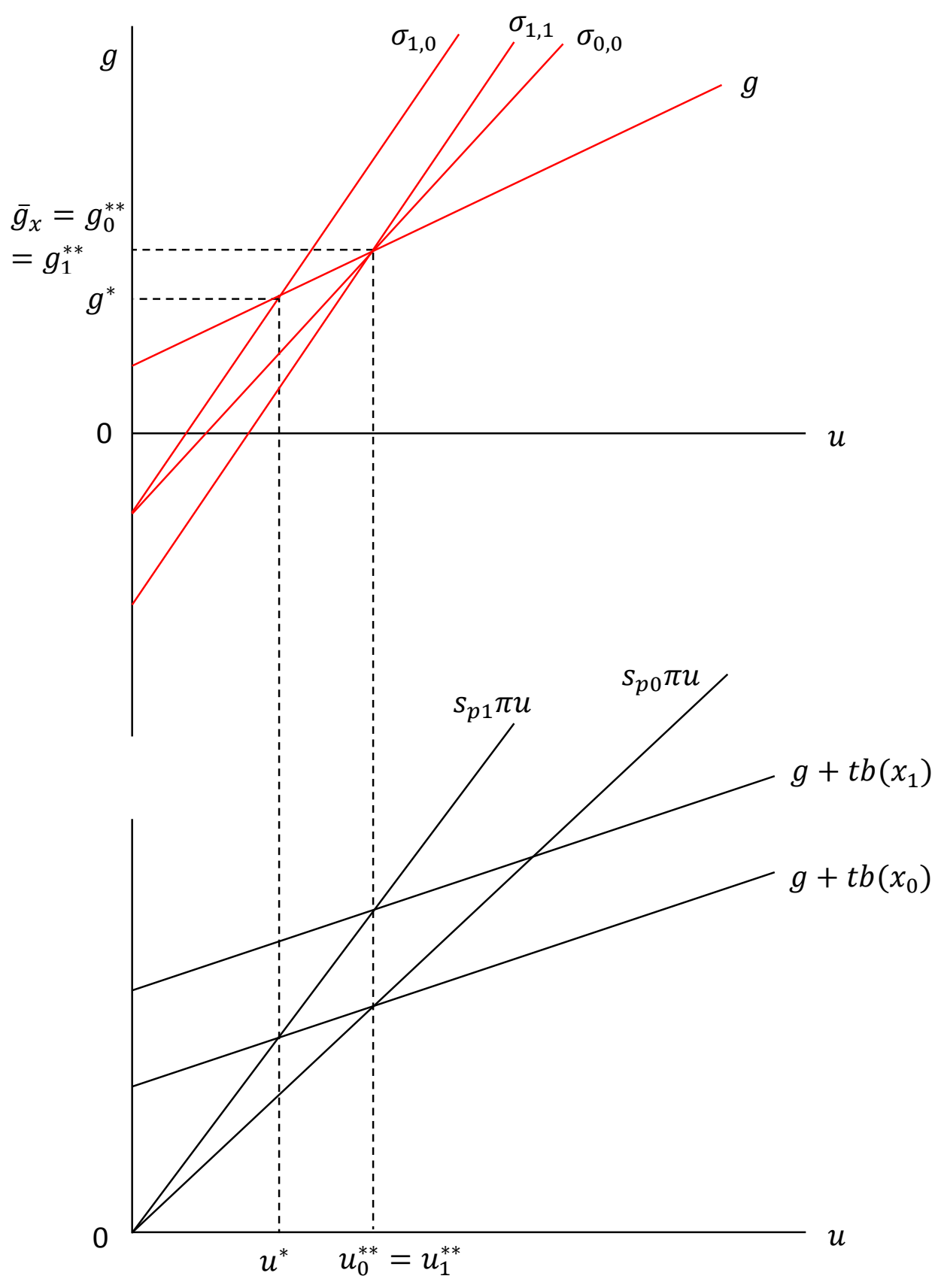

Figure 1. Short vs. medium run impacts of an increase in the propensity to save 


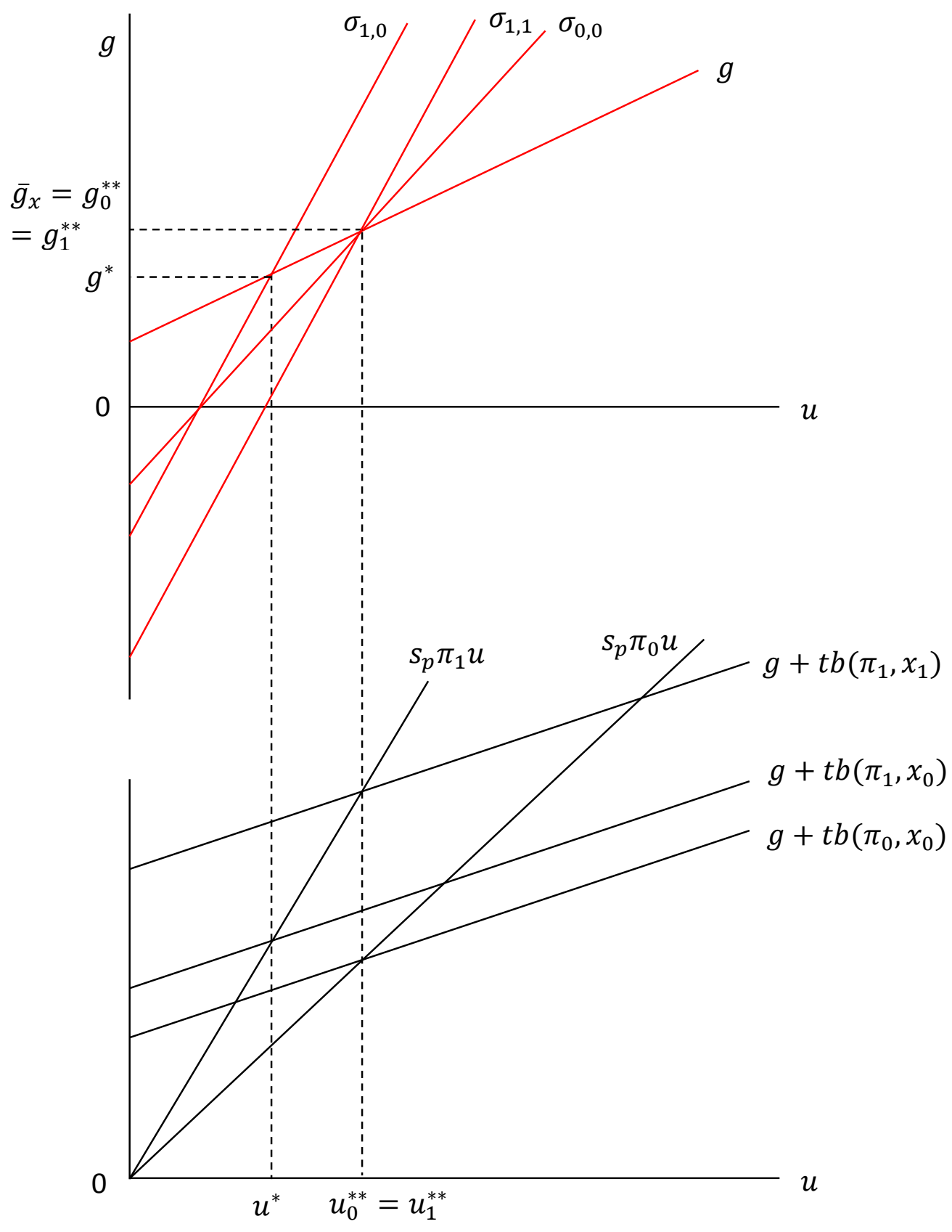

Figure 2. Short vs. medium run impacts of an increase in the share of profits 


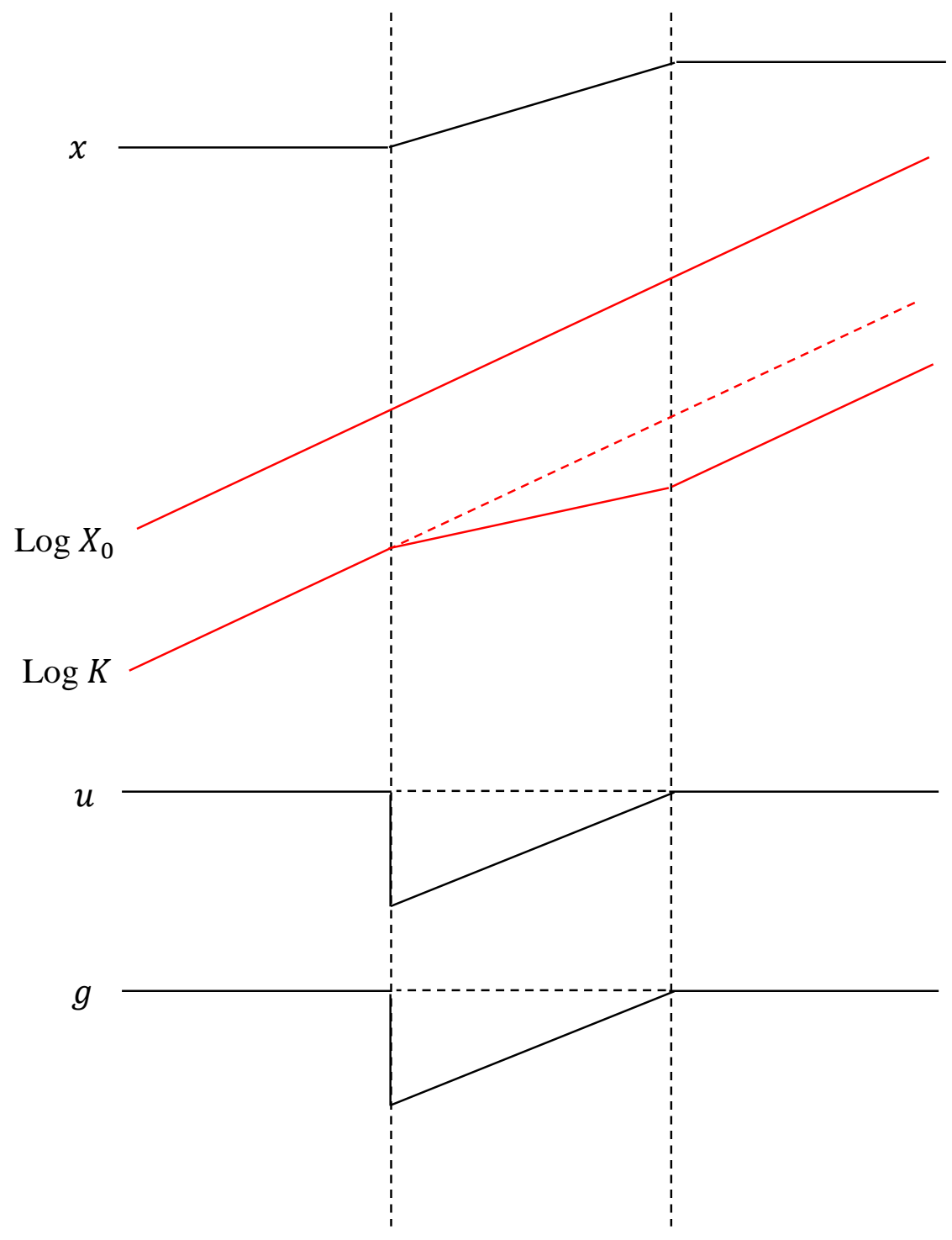

Figure 3. Short vs. medium run responses of endogenous variables after a permanent increase in the propensity to save, or in the share of profits 


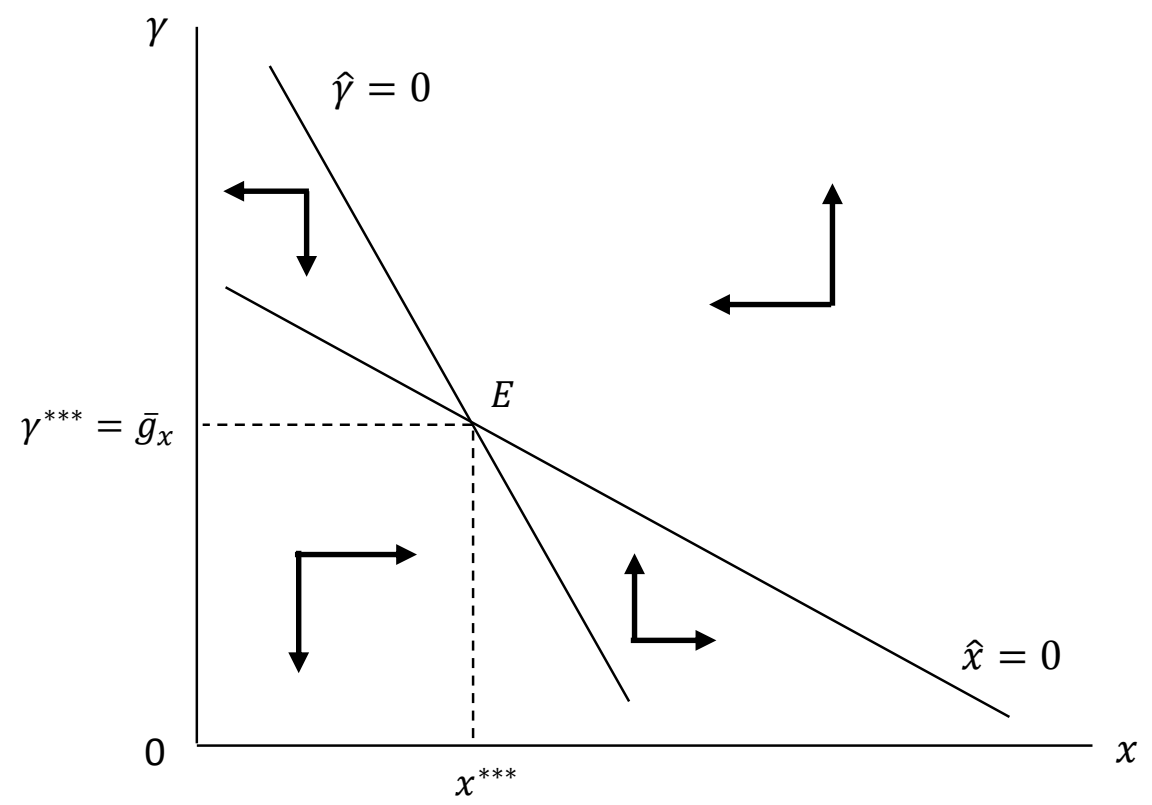

Figure A1. Phase diagram: Dynamics of $x$ and $\gamma$ 


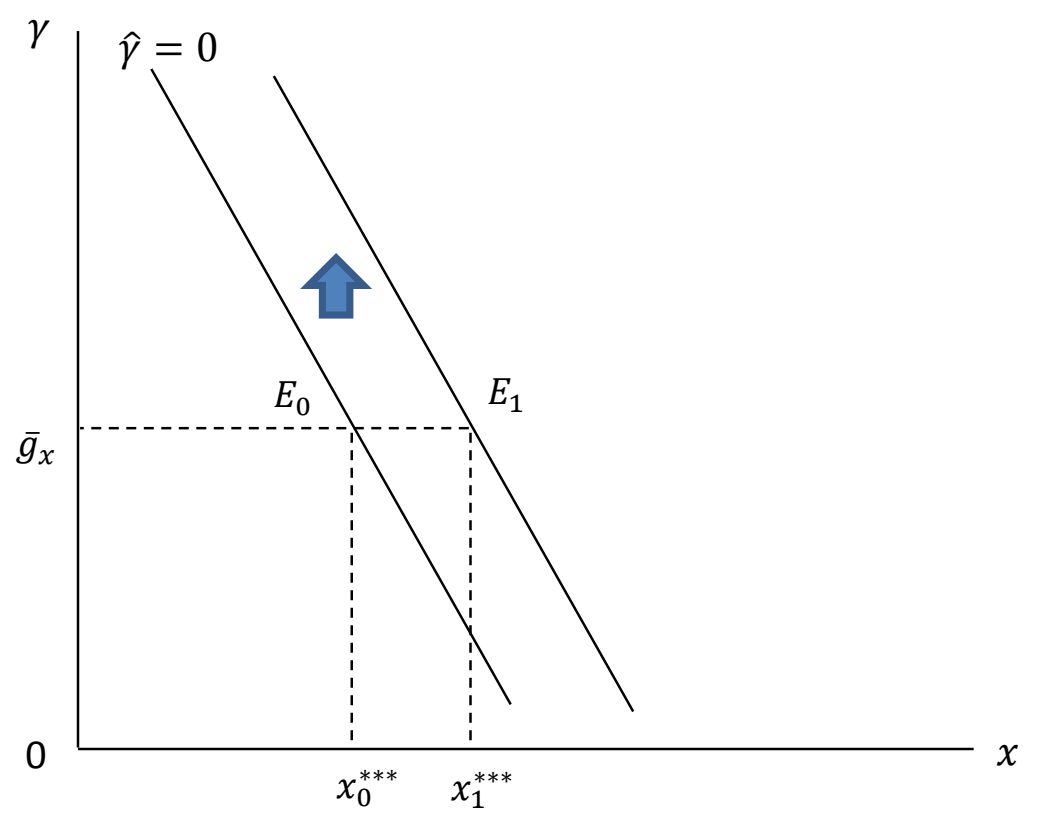

Figure A2. Long run effects of a permanent increase in the propensity to save, or in the share of profits 


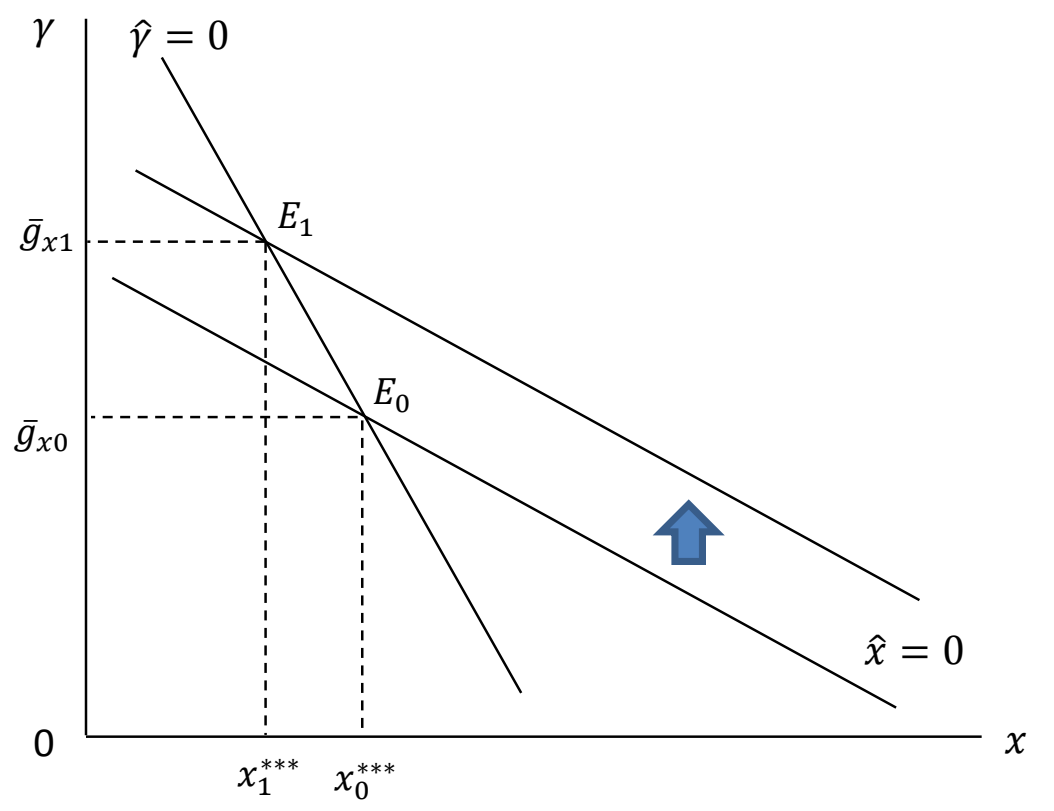

Figure A3. Long run effects of a permanent increase in the exogenous growth rate of autonomous exports 